\title{
Oxidação do enxofre elementar em solos tropicais
}

\author{
Oxidation of elemental sulfur in tropical soils
}

Nelson Horowitz ${ }^{1}$ Egon José Meurer ${ }^{2}$

\section{RESUMO}

O uso de fertilizantes que contêm baixos teores de enxofre (S), em solos com baixos teores de matéria orgânica, pode resultar em limitação desse nutriente para as culturas. $O$ uso do S-elementar como fertilizante, isoladamente ou associado a fórmulas comerciais, pode reduzir os custos de adubação em solos deficientes de S. Os objetivos deste estudo foram: a) verificar se, em dois solos tropicais, ocorre oxidação de S-elementar; b) avaliar se o período de incubação de seis dias sugerido por Janzen \& Bettany (1987a) é adequado para estimar a oxidação do S-elementar e c) medir o efeito da oxidação do S-elementar sobre o $\mathrm{pH}$ do solo. Para isso, foi realizado um experimento com amostras de um ARGISSOLO VERMELHO AMARELO eutrófico abruptíco e de um LATOSSOLO VERMELHO DISTRÓFICO típico. As amostras dos dois solos foram incubadas com seis doses de enxofre (0, 1,5, 3,0, 6,0 9,0 e $12 \mathrm{~g}$ de $\mathrm{S}^{\circ} \mathrm{kg}^{-1}$ de solo) por períodos entre 0 e 70 dias. A incubação foi feita em estufa, a uma temperatura de $27^{\circ} \pm 1^{\circ} \mathrm{C}$. Ambos os solos tiveram capacidade para oxidar o S-elementar para S-sulfato, forma disponível à planta. No argissolo, o teor de $S$-sulfato resultante da oxidação do $S$ elementar foi $563 \mathrm{mg} \mathrm{dm}^{-3}$ de solo, enquanto que, no latossolo, foi de $207 \mathrm{mg} \mathrm{dm}^{-3}$ de solo, indicando capacidade diferenciada de ambos para oxidar o S-elementar. Em ambos os solos houve diminuição do $\mathrm{pH}$, sendo mais acentuada para o argissolo. $O$ período de incubação para avaliação da oxidação do $S$ elementar de seis dias sugerido por Janzen \& Bettany (1987a) parece ser insuficiente.

Palavras-chave: sulfato, fertilizante, incubação, capacidade de oxidação, $p H$.

\section{ABSTRACT}

The use of fertilizers with low content of sulfur (S), in low organic matter content soil, may result in sulfur limitation to crops. The use of elemental-S as a fertilizer, isolated or associated to commercial formula, may reduce the costs of fertilization in sulfur deficient soils. The objectives of this study were: a) to verify if two tropical soils have capacity to oxidize elemental-S; b) to evaluate if the period of incubation suggested by Janzen \& Bettany (1987a) is adequate to estimate the elemental-S oxidation and c) to measure the effect on soil $\mathrm{pH}$ of the added elemental-S. For this, a trial was carried out with soil samples of an Typic Hapludalf and a Typic Haplustox (SOIL SURVEY STAFF, 1999). The samples of both soils were incubated with dosis of sulphur $(0,1,5,3,0,6,0,9,0$, and $12 \mathrm{mg}$ of $S^{0} \mathrm{~kg}^{-1}$ of soil), for periods between 0 and 70 days. The temperature of incubation was $27^{\circ} \pm 1^{\circ} \mathrm{C}$. Both soils showed capacity of oxidizing the applied elemental $S$ to $S$-sulfate, available form to plant. In the Ultisol oxidation of elemental$S$ produced maximum values of 563 of $S$-sulfate $\mathrm{mg} \mathrm{dm}^{-3}$ of soil and in the Oxisol soil 207 of S-sulfate $\mathrm{mg} \mathrm{dm}^{-3}$ of soil, demonstrating the differentiated capacity of elemental-S oxidation. In both soils the $\mathrm{pH}$ decreased, being the diminution bigger for the Ultisol. The incubation period of six days for the evaluation of the elemental-S oxidation suggested by Janzen \& Bettany seems to be insufficient.

Key words: sulphate, fertilizer, incubation, oxidation capacity, $p H$.

\section{INTRODUÇÃO}

Deficiências de enxofre ocorrem em diversas regiões do Brasil, notadamente nas áreas dos Cerrados. As fontes mais utilizadas para suprir o $S$ às plantas são o superfosfato simples, que contém $12 \%$ de S-sulfato, e o sulfato de amônio, com $24 \%$ de S-sulfato. Ambos são utilizados isoladamente ou como componentes de fertilizantes comerciais. Autilização de S-elementar, que contém mais de $90 \%$ de $\mathrm{S}$, incorporado a fertilizantes comerciais é uma alternativa que pode aumentar a concentração dos nutrientes nas formulações e reduzir os custos de produção, de transporte e de aplicação do fertilizante.

${ }^{1}$ Roullier Brasil, Porto Alegre, RS, Brasil. E-mail: nelsontz@terra.com.br. Autor para correspondência.

${ }^{2}$ Departamento de Solos da Universidade Federal do Rio Grande do Sul (UFRGS), Porto Alegre, RS, Brasil. E-mail egon.meurer@ufrgs.br. 
O S-elementar aplicado ao solo deve ser oxidado para S-sulfato para ser absorvido pelas plantas (JANZEN \& BETTANY, 1987a). A oxidação pode ser realizada por fatores abióticos; porém, as reações catalisadas pelos microrganismos são os principais agentes nesse processo. Fatores de solo como $\mathrm{pH}$, textura, disponibilidade de nutrientes, aeração e temperatura podem afetar a oxidação do S-elementar a S-sulfato(GERMIDA \& JANZEN, 1993). O pH do solo é, quase sempre, relacionado com a taxa de oxidação do S-elementar (NOR \& TABATABAI, 1977; JANZEN \& BETTANY, 1987b; LAWRENCE \& GERMIDA, 1988). $\mathrm{O}$ efeito do $\mathrm{pH}$ na oxidação do S-elementar possivelmente está relacionado à capacidade do solo em tamponar o ácido sulfúrico formado na oxidação, que, se acumulado em altas concentrações, inibe a atividade dos microrganismos que transformam Selementar em S-sulfato (FOX et al.,1964; BARROW, 1971).

Existem alguns métodos para determinar a taxa de oxidação do S-elementar em solos: a) os que determinam o S-sulfato acumulado após seis dias de incubação do solo com S-elementar (JANZEN \& BETTANY 1987a; TABATABAI, 1993) e b) métodos que determinam o S-elementar remanescente após 70 dias ou mais de incubação, como proposto por WATKINSON (1989). Os métodos que sugerem que a incubação seja feita por seis dias são contestados, pois, segundo WATKINSON (1989), é provável que este período seja curto para a estimativa correta das taxas de oxidação do enxofre elementar. Esse autor sugere que os solos sejam incubados com o S-elementar por, no mínimo, 70 dias. Outra discordância entre as metodologias é a respeito da quantidade de S-elementar que deve ser aplicada ao solo que será incubado. As quantidades recomendadas variam de 0,5 a 10 gramas de S-elementar por kg de solo seco. É possível que, com a adição de maiores quantidades de S-elementar, ocorra inibição da oxidação microbiológica devido à liberação de substâncias tóxicas em teores elevados (JANZEN \& BETANNY, 1987a). Isto poderia diminuir significativamente a taxa de oxidação, prejudicando a sua medição.

Há poucos dados de pesquisa sobre a capacidade dos solos brasileiros em oxidar o Selementar e os métodos descritos na literatura apresentam divergências. Assim, este estudo teve por objetivos: a) verificar se em dois solos tropicais ocorre oxidação de S-elementar; b) avaliar se o período de incubação de seis dias sugerido por Janzen \& Bettany (1987a) é adequado para estimar a oxidação do Selementar e c) medir o efeito da oxidação do S-elementar sobre o $\mathrm{pH}$ do solo.

\section{MATERIAL E MÉTODOS}

O estudo foi conduzido nos laboratórios do Departamento de Solos da Faculdade de Agronomia da Universidade Federal do Rio Grande do Sul, entre dezembro de 2000 e março de 2001. Constou de um experimento realizado com amostras de dois solos com características químicas e físicas contrastantes (teor de argila, $\mathrm{pH}$, teor de $\mathrm{P}$ e matéria orgânica): um ARGISSOLO VERMELHO AMARELO eutrófico abruptíco e de um LATOSSOLO VERMELHO DISTRÓFICO típico (EMBRAPA, 1999). A amostra do latossolo foi coletada em área nunca cultivada, na Embrapa-Cerrados, localizada em Planaltina, Distrito Federal. A amostra do argissolo foi coletada em Pindorama, estado de São Paulo, de uma parcela experimental que vem sendo cultivada com culturas anuais. As amostras coletadas no horizonte A, na profundidade de $0 \mathrm{a} 20 \mathrm{~cm}$, foram secados à sombra e tamisadas em peneiras com malhas de 2,0mm de abertura. Na tabela 1, encontram-se os resultados das análises de alguns atributos físicos e químicos das amostras dos dois solos determinados conforme métodos descritos por TEDESCO et al., 1995.

Os tratamentos consistiram na aplicação Selementar nas doses de 0; 1,5; 3; 6; 9 e $12 \mathrm{~g} \mathrm{~kg}^{-1}$ de solo seco, que foram incubados por períodos de $0,6,22,36$, 54 e 70 dias. O tamanho das partículas do S-elementar misturadas às amostras dos solos situou-se entre $0,074 \mathrm{~mm}$ e $0,15 \mathrm{~mm}$. O S-elementar que foi misturado às amostras dos dois solos foi previamente peneirado com etanol, que é um dispersante de S-elementar. Este procedimento assegura a remoção de partículas finas aderidas às partículas do tamanho desejado (JANZEN \& BETTANY, 1987a). Depois desta tamização em meio líquido, o S-elementar foi secado em estufa a $65^{\circ} \mathrm{C}$ e, a seguir, misturado com as amostras dos solos secos que foram umedecidas até $80 \%$ da capacidade de retenção de água e acondicionadas em frascos de vidro com capacidade para $150 \mathrm{~mL}$. Os frascos foram fechados com tampas plásticas perfuradas para permitir a condição de aerobiose. Os frascos foram colocados em estufa com temperatura regulada para $27^{\circ} \pm 1^{\circ} \mathrm{C}$. A cada sete dias, a umidade foi medida e restabelecida a quantidade inicial de água por meio de uma seringa, introduzindo-se a agulha em vários pontos do solo, depositando-se assim a água, sem modificar a acomodação e a estrutura das amostras dentro dos frascos. Cada tratamento foi realizado com três repetições, totalizando 216 frascos. Após a retirada dos frascos da estufa, em cada período de incubação, as amostras dos solos foram secadas em outra estufa, com circulação forçada de ar, ajustada para $40^{\circ} \mathrm{C}$. Após 
Tabela 1 - Alguns atributos das amostras do argisssolo e do latossolo coletadas no horizonte A, na profundidade de 0 a $20 \mathrm{~cm}$ de profundidade, nos municípios de Pindorama (SP) e Planaltina (DF), respectivamente.

\begin{tabular}{|c|c|c|c|c|c|c|c|c|c|c|c|c|c|}
\hline Solos & $\begin{array}{c}\mathrm{PH} \\
\mathrm{H}_{2} \mathrm{O} \\
(1)\end{array}$ & $\begin{array}{c}\text { PH } \\
\text { SMP } \\
(2)\end{array}$ & $\begin{array}{c}\text { Argila } \\
\text { (3) }\end{array}$ & $\begin{array}{c}\text { Matéria } \\
\text { Orgânica } \\
\text { (4) }\end{array}$ & $\begin{array}{c}\text { P } \\
\text { (5) }\end{array}$ & $\mathrm{K}(5)$ & $\begin{array}{c}\mathrm{S}-\mathrm{SO}_{4} \\
(6)\end{array}$ & $\begin{array}{l}\mathrm{Ca} \\
(7)\end{array}$ & $\begin{array}{l}\mathrm{Mg} \\
(7)\end{array}$ & $\mathrm{Al}(7)$ & $\begin{array}{l}\mathrm{Cu} \\
(8)\end{array}$ & $\begin{array}{l}\mathrm{Mn} \\
\text { (7) }\end{array}$ & $\begin{array}{l}\mathrm{Zn} \\
\text { (8) }\end{array}$ \\
\hline & & \multicolumn{4}{|c|}{$\mathrm{g} \mathrm{kg}^{-1}$} & \multicolumn{2}{|c|}{$\mathrm{mg} \mathrm{dm} \mathrm{m}^{-3}$} & \multicolumn{3}{|c|}{$\mathrm{cmol}_{\mathrm{c}} \mathrm{dm}^{-3}$} & \multicolumn{3}{|c|}{$\mathrm{mg} \mathrm{dm} \mathrm{m}^{-3}$} \\
\hline argissolo & 6,4 & 6,8 & 100 & 11 & 47 & 93 & 6,5 & 3,0 & 1,3 & 0,0 & 2,4 & 5,0 & 2,5 \\
\hline latossolo & 4,2 & 5,1 & 470 & 44 & 1,6 & 27 & 4,5 & 0,6 & 0,2 & 1,6 & 0,5 & 6,0 & 0,6 \\
\hline
\end{tabular}

(1) relação solo:água destilada 1:1; (2) solução tamponada a pH 7,5; (3) densímetro; (4) Walkley-Black; (5) Melich-1; (6) Fosfato de cálcio; (7) $\mathrm{KCl} 1 \mathrm{~mol} \mathrm{~L}^{-1}$; (8) $\mathrm{HCl} 0,1 \mathrm{~mol} \mathrm{~L}^{-1}$

a secagem, foram determinados o pH em água e o teor de S-sulfato por extração com fosfato de cálcio, conforme os métodos descritos por TEDESCO et al. (1995).

A análise da variância para os resultados obtidos foi realizada de acordo com o seguinte modelo: $\mathrm{Y}_{\mathrm{ijk}}=\mu+\alpha_{\mathrm{i}}+\beta_{\mathrm{j}}+\alpha \beta_{\mathrm{ij}}+\varepsilon_{\mathrm{ijk}}$ onde, $\mathrm{i}=1,2$ (índice de solos); $\mathrm{j}=1,2, \ldots 7$ (índice de dias de incubação) e $\mathrm{k}=1,2,3$ (índice de repetição), sendo $\mathrm{Y}_{\mathrm{ijk}}=\mathrm{a}$ observação da variável avaliada na repetição k da combinação do nível i de solo com o nível j de dias de incubação; $\mu=$ média geral; $\alpha_{i}=$ efeito de solo; $\beta=$ efeito de dias de incubação; $\alpha \beta_{\mathrm{ij}}=$ efeito da interação entre solo e dias de incubação e $\varepsilon_{\mathrm{ijk}}=$ erro aleatório não-observável associado à observação.

\section{RESULTADOS E DISCUSSÃO}

Tanto no argissolo como no latossolo ocorreu a oxidação do S-elementar para S-sulfato (Figuras 1 e 2). Após os 70 dias de incubação, as quantidades de S-sulfato no argissolo foram acentuadamente superiores do que aquelas obtidas no latossolo. No primeiro solo, a quantidade máxima de Ssulfato determinada foi de $563 \mathrm{mg} \mathrm{dm}^{-3}$, enquanto que no latossolo foi de $207 \mathrm{mg} \mathrm{dm}^{-3}$ de solo. Em ambos os solos, no tratamento em que não houve a adição de Selementar (testemunha), o teor de S-sulfato, durante o período de 70 dias de incubação, não apresentou alteração significativa.

Nos dois solos, somente a partir do 22ํ dia de incubação houve um aumento significativo da quantidade de S-sulfato. No argissolo, o aumento foi praticamente linear até o 54ํㅡㄹ dia para todas as doses de S-elementar aplicadas (Figura 1). A partir desta data, para a dose de $9 \mathrm{~g} \mathrm{~S}^{0} \mathrm{~kg}^{-1}$ não houve acréscimo significativo no teor de S-sulfato. Para a dose mais elevada de $12 \mathrm{~g} \mathrm{~S}^{0} \mathrm{~kg}^{-1}$, houve uma redução do S-sulfato no solo de $510 \mathrm{mg} \mathrm{dm}^{-3}$ para $450 \mathrm{mg} \mathrm{dm}^{-3}$. No argissolo, a maior quantidade de S-sulfato foi obtida com a dose aplicada de $3 \mathrm{~g} \mathrm{~S}^{0} \mathrm{~kg}^{-1}$, aos 70 dias de incubação. Nesta dose, o teor de S-sulfato no argissolo foi crescente em todos os períodos de incubação do solo com Selementar, indicando ser a dose de maior produção de S-sulfato.

No latossolo, o aumento da quantidade de S-sulfato foi linear até o $70^{\circ}$ dia para todas as doses aplicadas (Figura 2). Entretanto, na dose de $12 \mathrm{~g} \mathrm{~S}^{0} \mathrm{~kg}^{-1}$, a quantidade de S-sulfato foi de $180 \mathrm{mg} \mathrm{dm}^{-3}$, sendo inferior às demais doses. Nesse solo, similarmente ao que ocorreu no argissolo, a maior quantidade de Ssulfato foi verificada para a dose de $3 \mathrm{~g} \mathrm{~S}^{0} \mathrm{~kg}^{-1}$, aos 70 dias da incubação.

Os valores do pH em água, medidos durante os períodos de incubação nos dois solos, encontramse na tabela 2 . O pH dos solos relaciona-se, geralmente, com a oxidação do S-elementar catalizada pelos microrganismos (NOR \& TABATABAI, 1977; LAWRENCE \& GERMIDA, 1988). No argissolo, o pH decresceu à medida que aumentaram os períodos de incubação, atingindo o valor de 3,3, cerca de 2,8 unidades abaixo do valor original. No latossolo, entretanto, a oxidação do S-elementar, após os 70 dias de incubação, resultou em mudanças menores no $\mathrm{pH}$, com variação máxima de valores de 0,4 unidades. A menor variação de $\mathrm{pH}$ ocorrida no latossolo, em comparação à ocorrida para o argissolo, está relacionada ao maior poder tampão do latossolo, que tem maiores teores de argila e de matéria orgânica.

Os resultados obtidos na análise estatística mostraram que o teor de S-sulfato no solo depende da interação solo $\mathrm{x}$ doses de S-elementar $\mathrm{x}$ dias de incubação $(\mathrm{P}<0,0001)$. Isso indica que o período de incubação tem efeito na quantidade convertida de Selementar para S-sulfato e depende do tipo de solo e da dose de S-elementar utilizada. Os resultados de Ssulfato obtidos nos dois solos, no período de 70 dias, e as alterações no $\mathrm{pH}$ do solo indicam que o argissolo 


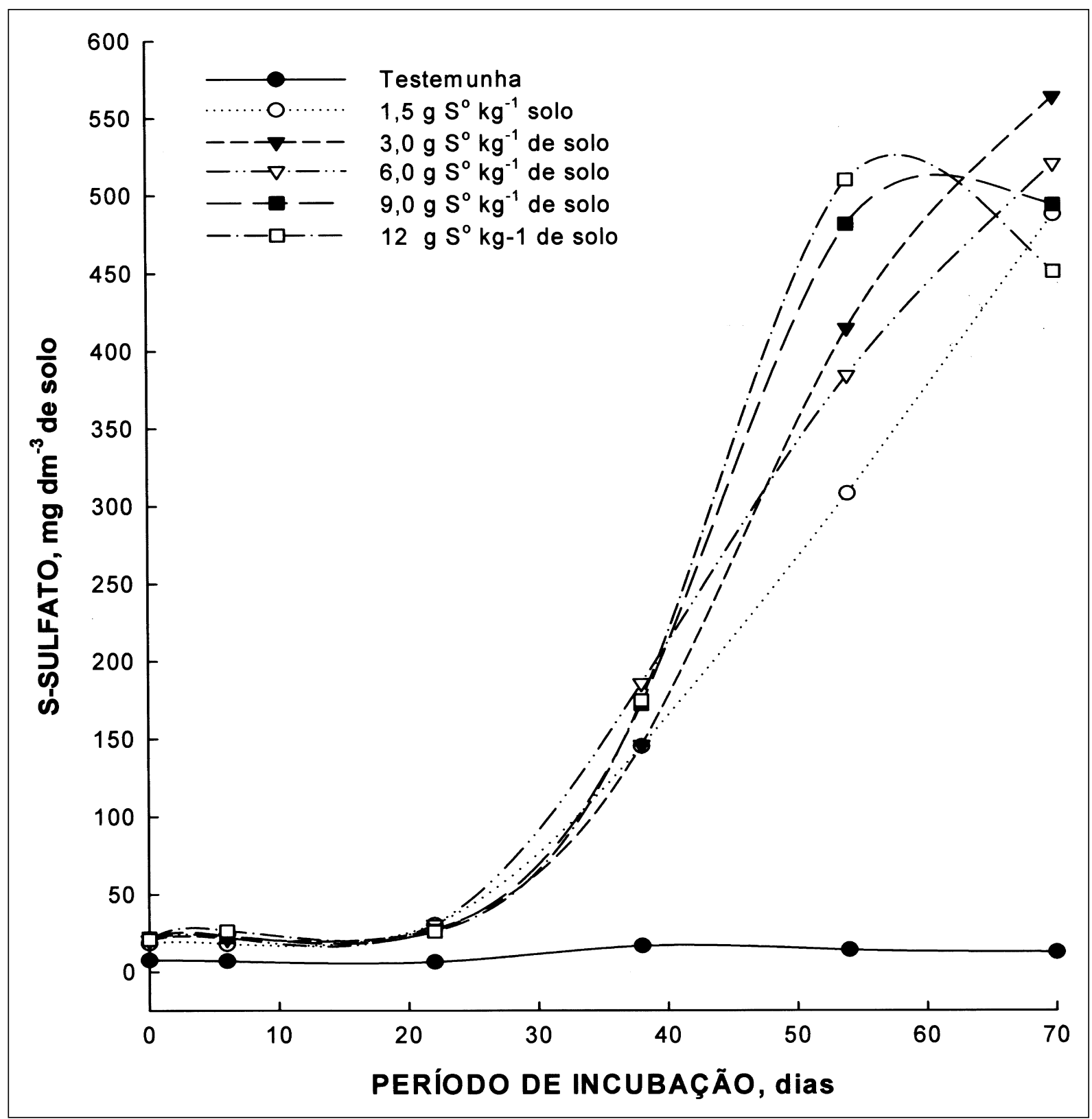

Figura 1 - Teor de S-sulfato no argissolo em função dos períodos de incubação para doses de S-elementar adicionadas ao solo.

apresentou, provavelmente, uma maior capacidade de oxidar o S-elementar quando comparado ao latossolo, uma vez que a quantidade de S- sulfato no argissolo foi de $563 \mathrm{mg} \mathrm{dm}^{-3}$, ou seja, 2,72 vezes maior que a quantidade obtida no latossolo. É possível que isso tenha ocorrido devido às melhores condições de fertilidade do argissolo, principalmente devido ao valor do $\mathrm{pH}$ inicial e o teor de fósforo mais elevados (Tabela 1). A textura do argissolo, com maior teor de areia, pode ter contribuído para esses resultados, concordando com a afirmação de que a conversão de S-elementar em
S-sulfato é inversamente relacionada ao teor de argila do solo (JANZEN \& BETTANY, 1987c; LAWRENCE \& GERMIDA, 1988; DENG \& DICK, 1990). Por outro lado, o maior teor de matéria orgânica do latossolo $\left(44 \mathrm{~g} \mathrm{~kg}^{-1}\right)$ em relação ao do argissolo $\left(11 \mathrm{~g} \mathrm{~kg}^{-1}\right)$ não foi um fator que individualmente afetou a oxidação do S-elementar. Isso sugere que a interação de vários fatores é mais importante do que a ação de cada um isoladamente (GERMIDA \& JANZEN, 1993).

Quanto ao efeito da dose de S-elementar utilizada na incubação, que variou de zero até $12 \mathrm{~g} \mathrm{~kg}^{-1}$, 


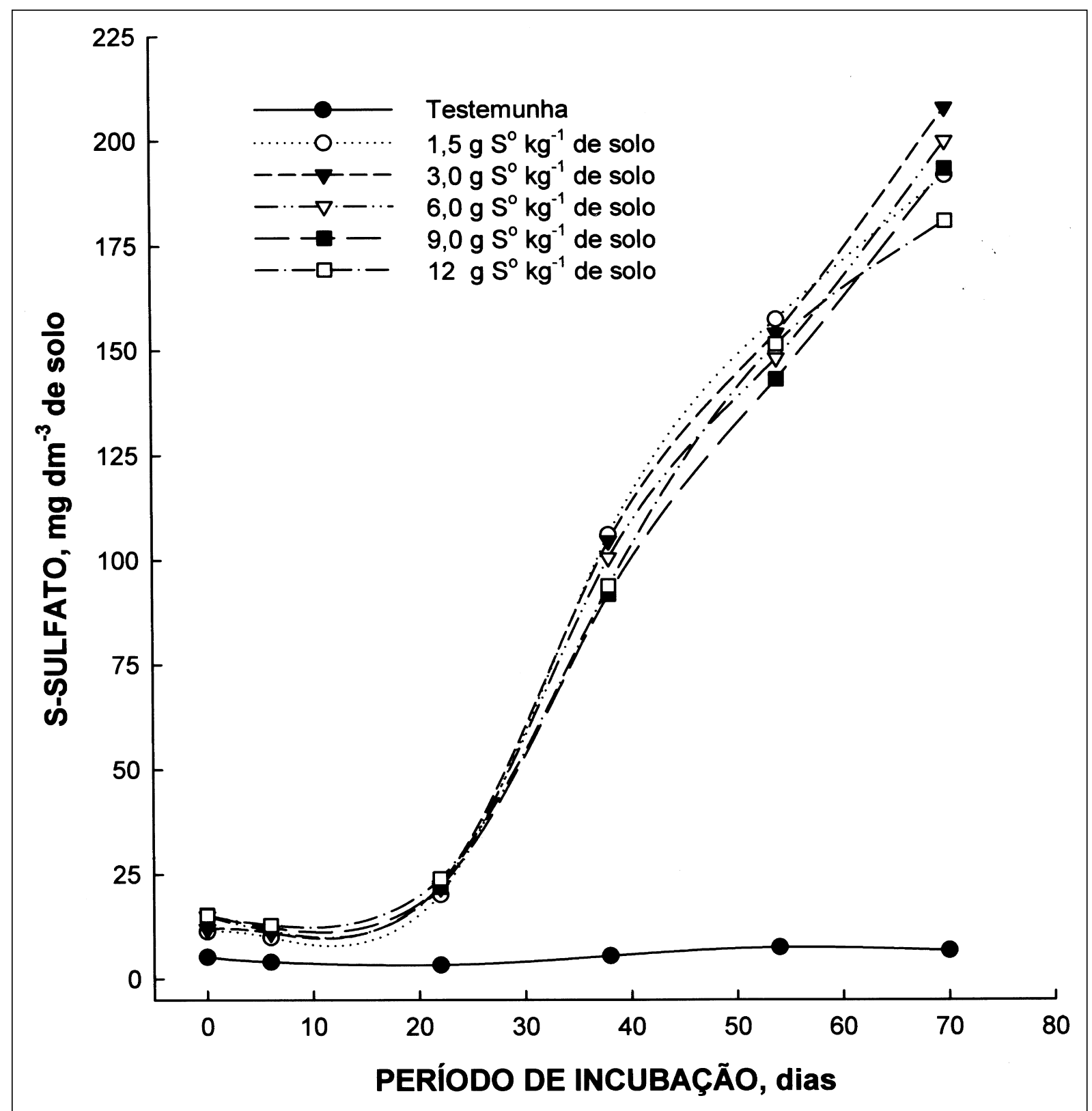

Figura 2 - Teor de S-sulfato no latossolo em função dos períodos de incubação para doses de S-elementar adicionadas ao solo.

observou-se, principalmente no argissolo, que, na maior dose, pode ter ocorrido um possível efeito inibitório na oxidação do S-elementar. Isso teria ocorrido devido ao acúmulo de produtos da oxidação, tóxicos ou ácidos, os quais podem ter inibido a conversão do S-elementar para S-sulfato. Por outro lado, os resultados mostraram (Figuras 1 e 2) que doses maiores do que $3 g \mathrm{~S}^{0} \mathrm{~kg}^{-1} \mathrm{de}$ solo, para os períodos de incubação utilizados, não resultaram em maiores quantidades de S-sulfato em ambos os solos. Esses resultados indicam que pode existir um limite para a dose de S-elementar a ser adicionada ao solo, a partir do qual pode ocorrer a inibição da oxidação do S-elementar pelo acúmulo de produtos ácidos e tóxicos aos microrganismos.

Em relação ao período de incubação, que variou de zero a 70 dias, observou-se (Figuras 1 e 2) que, após o $22^{\circ}$ dia, a oxidação do S-elementar aumentou acentuadamente. Esses resultados estão provavelmente relacionados ao tempo de crescimento da população de microrganismos oxidantes e à colonização das partículas do S-elementar por estes microrganismos. Essa hipótese concorda com as 
Tabela 2 - Valor do pH em água, no período de incubação, em função das doses de S-elementar adicionadas ao argissolo e ao latossolo.

\begin{tabular}{|c|c|c|c|c|c|c|}
\hline \multirow{2}{*}{$\begin{array}{l}\text { Dose } \\
\left(\mathrm{g} \mathrm{S}^{0} \mathrm{~kg}^{-1}\right)\end{array}$} & \multicolumn{6}{|c|}{ Dias de incubação } \\
\hline & 0 & 6 & 22 & 38 & 54 & 70 \\
\hline & \multicolumn{6}{|c|}{ argissolo } \\
\hline 0 & 6,2 & 5,9 & 6,1 & 6,2 & 5,8 & 6,0 \\
\hline 1,5 & 6,2 & 6,0 & 5,6 & 5,0 & 4,4 & 4,0 \\
\hline 3 & 6,1 & 5,9 & 5,5 & 4,6 & 4,0 & 3,4 \\
\hline 6 & 6,1 & 5,9 & 5,1 & 4,3 & 3,8 & 3,3 \\
\hline 9 & 6,2 & 6,0 & 5,2 & 4,3 & 3,6 & 3,3 \\
\hline \multirow[t]{2}{*}{12} & 6,1 & 5,9 & 5,3 & 4,5 & 3,5 & 3,3 \\
\hline & \multicolumn{6}{|c|}{ latossolo } \\
\hline 0 & 4,2 & 4,2 & 3,9 & 4,0 & 3,8 & 3,8 \\
\hline 1,5 & 4,3 & 4,2 & 4,2 & 4,4 & 4,2 & 3,9 \\
\hline 3 & 4,2 & 4,2 & 4,3 & 4,4 & 4,2 & 3,9 \\
\hline 6 & 4,2 & 4,2 & 4,3 & 4,4 & 4,2 & 3,9 \\
\hline 9 & 4,2 & 4,2 & 4,3 & 4,4 & 4,3 & 4,0 \\
\hline 12 & 4,2 & 4,1 & 4,3 & 4,4 & 4,2 & 4,0 \\
\hline
\end{tabular}

observações de CHAPMAN (1989), que constatou que houve um período de defasagem de vários dias para o início do processo de oxidação em solos aos quais o Selementar havia sido adicionado.

Os teores de S-sulfato, em ambos os solos, até o sexto dia de incubação, foram praticamente os mesmos e corresponderam a cerca de $5 \%$ do total do Ssulfato no final do período de incubação de 70 dias. Esses resultados sugerem que o período de seis dias proposto por JANZEN \& BETTANY (1987a) e por TABATABAI (1993) para incubar solos com Selementar, com o objetivo de quantificar a taxa de oxidação, não é suficiente e concordam com a afirmação de WATKINSON (1989) de que o período de incubação deva ser maior.

\section{CONCLUSÕES}

O argissolo e o latossolo têm capacidade de oxidar S-elementar para S-sulfato. Os resultados indicaram que o período de incubação de seis dias é insuficiente para avaliar a oxidação do S-elementar, sugerindo que o método de Janzen \& Bettany não é adequado para estimar a oxidação do enxofre elementar nas amostras dos dois solos. A oxidação do Selementar para S-sulfato ocasionou diminuição do $\mathrm{pH}$ em ambos solos e foi mais acentuada no argissolo.

\section{AGRADECIMENTOS E APRESENTAÇÃO}

Ao Conselho Nacional de Desenvolvimento Científico e Tecnológico (CNPq), pela concessão de bolsa ao pesquisador Meurer.
Este trabalho é parte da Tese de Doutorado do primeiro autor submetida ao Programa de Pós-graduação em Ciência do Solo da Universidade Federal do Rio Grande do Sul, Porto Alegre, RS, Brasil.

\section{REFERÊNCIAS}

BARROW, N.J. Slowly available sulphur fertilizers in southwestern Australia. I.Elemental sulphur. Australian Journal of Experimental Agriculture and Animal Husbandry, Collingwood, v.2, p.211-216, 1971.

CHAPMAN, S.J. Oxidation of micronized elemental sulphur in soil. Plant and Soil, Dordrecht, v.116, p.69-76, 1989.

DENG, S.; DICK, R.P. Sulfur oxidation and rhodanese activity in soils. Soil Science, Baltimore, v.150, n.2, p.552-560, 1990.

EMBRAPA. Centro Nacional de Pesquisa de Solos. Sistema Brasileiro de Classificação de Solos. Brasília: Embrapa Produção de Informação; Rio de Janeiro: Embrapa Solos, 1999. 412p.

FOX, R.L. et al. Factors influencing the availability of sulfur fertilizers to alfafa and corn. Soil Science Society Proceedings, Madison, v.28, p.406-408, 1964.

GERMIDA, J.J.; JANZEN, H.H. Factors affecting the oxidation of elemental sulfur in soils. Fertilizer Research, Wageningen, Netherlands, v.35, p.101-114, 1993.

JANZEN, H.H.; BETTANY, J.R. Measurement of sulfur oxidation in sois. Soil Science, Baltimore, v.143, n.6, p.444452, 1987a.

JANZEN, H.H.; BETTANY, J.R. Oxidation of elemental sulfur under field conditions in Central Saskatchewan. Canadian Journal of Soil Science, Ottawa, v.67, p.609-618, 1987b. 
JANZEN, H.H.; BETTANY, J.R. The effect of temperature and water potential on sulfur oxidation in soils. Soil Science, Baltimore, v.144, n.2, p.81-89, 1987c.

LAWRENCE, J.R.; GERMIDA, J.J. Relationship between microbial biomass and elemental sulfur oxidation in agricultural soils. Soil Science Society of America Journal, Madison, v.52, p.672-677, 1988.

NOR, Y.M.; TABATABAI, M.A. Oxidation of elemental sulfur in soils. Soil Science Society of America Journal, Madison, v.41, p.736-741, 1977.

TABATABAI, M.A. Sulfur oxidation and reduction in soils. In: JACKSON, S. Methods of soil analysis. Part 2:
Microbiological and biochemical properties. Madison: SSSA, 1993. p.1067-1078. (SSSA Book Series, 5).

TEDESCO, M.J. et al. Análises de solo, plantas e outros materiais. 2.ed. Porto Alegre: Departamento de Solos da UFRGS, 1995. 174p. (Boletim Técnico de Solos 5).

WATKINSON, J.H. Measurement of the oxidation rate of elemental sulfur in soil. Australian Journal of Soil Research, Collingwood, v.27, p.365-375, 1989.

SOIL SURVEY STAFF. Soil taxonomy: a basic system of soil classification for making and interpreting soil surveys. 2.ed. Washington: US Department of Agriculture, 1999. 869p. 